\title{
Estimation of the magnitude of Quadrupole Relaxation Enhancement in the context of Magnetic Resonance Imaging contrast
}

\author{
Danuta Kruk*a Elzbieta Masiewicz ${ }^{\mathrm{a}}$, Evrim Umut ${ }^{\mathrm{a}}$, Andreas Petrovic ${ }^{\mathrm{b}}$, Rupert Kargl ${ }^{\mathrm{b}, \mathrm{c}}$, \\ Hermann Scharffeter ${ }^{\mathrm{d}}$
}

\begin{abstract}
${ }^{a}$ Faculty of Mathematics and Computer Science, University of Warmia \& Mazury in Olsztyn, Słoneczna 54, 10-710 Olsztyn, Poland.

b Institute for Chemistry and Technology of Materials, Graz University of Technology, Stremayrgasse 9, 8010 Graz, Austria

${ }^{\mathrm{c}}$ Laboratory for Characterization and Processing of Polymers (LCPP), Faculty of Mechanical Engineering, University of Maribor, Smetanova ulica 17, 2000 Maribor, Slovenia

${ }^{d}$ Institute of Medical Engineering, Graz University of Technology, Stremayrgasse 16 / III, A8010 Graz, Austria.
\end{abstract}

*Corresponding author:

Danuta Kruk

e-mail: danuta.kruk@matman.uwm.edu.pl

Phone: +48 895246011 


\begin{abstract}
Magnetic Resonance Imaging (MRI) is one of the most powerful diagnostic tools providing maps of ${ }^{1} \mathrm{H}$ relaxation times of human body. The method needs, however, a contrast mechanism to enlarge the difference in the relaxation times between healthy and pathological tissues. In this work we discuss the potential of a novel contrast mechanism for MRI, based on Quadrupole Relaxation Enhancement (QRE) and estimate the achievable value of QRE under the most favorable conditions. It has turned out that the theoreticaly possible enhancement factors are smaller than those of typical paramagnetic contrast agents but in turn the field-selectivity of QRE-based agents makes them extremely sensitive to subtle changes of the electric field gradient in the tissue.

So far QRE has been observed for solids (in most cases for ${ }^{14} \mathrm{~N}$ ) as a result of very slow dynamics and anisotropic spin interactions, believed to be necessary for QRE to appear. We show the first evidence that QRE can be achieved in solutions of compounds containing a high spin nucleus $\left({ }^{209} \mathrm{Bi}\right)$ as the quadrupole element. The finding of QRE in liquid state is explained in terms of spin relaxation theory based on the stochastic Liouville equation. The results confirm the relaxation theory and motivate further exploration of the potential of QRE for MRI.
\end{abstract}




\section{INTRODUCTION}

Magnetic Resonance Imaging (MRI) provides spatially and temporally resolved maps of ${ }^{1} \mathrm{H}$ relaxation times of the inside of human bodies. The need to enlarge the difference (contrast) in the relaxation times between healthy and pathological tissues has given rise to a development of paramagnetic contrast agents causing Paramagnetic Relaxation Enhancement (PRE) effects. ${ }^{1-3}$ Paramagnetic contrast agents are complexes of transition or rare-earth metal ions. Usually Gadolinium based chelates are used in clinical practice. ${ }^{3-7}$ The ${ }^{1} \mathrm{H}$ relaxation enhancement is achieved due to very strong magnetic electron-proton dipole-dipole interactions between the electron spin of the paramagnetic species and neighbouring proton spins. As the ratio between the electron and proton gyromagnetic factors is very large (about 656 times larger than the proton gyromagnetic factor) and the ${ }^{1} \mathrm{H}$ relaxation rate depends on a square of the electron gyromagnetic factor, one could expect a very large PRE. In practice, the observed ${ }^{1} \mathrm{H}$ relaxation enhancement is much smaller (at least at lower magnetic fields) due to fast electron spin relaxation (caused by Zero Field Splitting interactions) that acts as an additional (besides the molecular motion) source of modulations of the electron-proton dipole-dipole interaction. ${ }^{1,2,8-15}$

In this work we demonstrate the prospective of a novel contrast mechanism for MRI, referred to as Quadrupole Relaxation Enhancement (QRE). ${ }^{16-25} \mathrm{QRE}$ is a complex, quantummechanical phenomenon which, in the context of MRI, is sometimes regarded as a counterpart of PRE, although this analogy is not fully justified. QRE leads to a frequency specific relaxation of ${ }^{1} \mathrm{H}$ spin-lattice relaxation (referred to as quadrupole peaks) originating from dipole-dipole interactions between protons $\left({ }^{1} \mathrm{H}\right.$ nuclei) and nuclei possessing a quadrupole moment. As a potential alternative to PRE this approach opens several desirable possibilities which are interesting in terms of molecular imaging with MRI. Although there is a clear theoretical foundation and the effect has been shown for ${ }^{14} \mathrm{~N}$ of the amide groups of proteins in low-field MRI experiments, ${ }^{18}$ no attempts have been made so far to exploit QRE for extrinsic MRI contrast agents. In this context it is worth to point out that actually the results available for solids are also mostly limited to ${ }^{14} \mathrm{~N} .{ }^{16-24}$ Considering QRE as a mechanism of novel extrinsic MRI contrast agents it is very important to stress that while PRE is observed in the whole frequency range as a result of a strong proton-electron dipole-dipole coupling, in the case of QRE the ${ }^{1} \mathrm{H}$ relaxation enhancement appears only at selected frequencies. The frequency positions of the QRE peaks can be altered by subtle changes in the EFG offering the possibility 
to activate or inactivate QRE contrast. This offers a high potential for the design of frequency selective MRI contrast agents in the context of molecular imaging.

To our knowledge so far there are no literature reports showing QRE effects for liquids (solutions). Looking for promising quadrupole nuclei (nuclei possessing a quadrupole moment) we have selected ${ }^{209} \mathrm{Bi}$ as a candidate as it fulfils a series of conditions: high gyromagnetic ratio and high spin quantum number ( $S=9 / 2$ ) to provide a strong $I-S$ dipole-dipole coupling, a large quadrupole moment that opens the opportunity to observe QRE effects at magnetic fields of $1.5 \mathrm{~T}$ or $3 \mathrm{~T}$ used in many clinical MRI scanners, ${ }^{25,26}$ relatively low toxicity, high natural abundance and rich chemistry that allows to vary the structure of ${ }^{209} \mathrm{Bi}$ containing compounds. The aim of this paper is to estimate the achievable amplitude of the QRE and show a first evidence that QRE can be observed in solutions of compounds containing ${ }^{209} \mathrm{Bi}$ as quadrupole element and hence motivate the further exploration of the potential of QRE for MRI.

\section{QUADRUPOLE RELAXATION ENHANCEMENT AND POLARIZATION TRANSFER}

The mechanism of QRE is simpler for solids than for liquids due to very slow dynamics of solid state systems. The QRE effect includes at least one nucleus of spin quantum number $I=1 / 2$ (for instance ${ }^{1} \mathrm{H}$ ) and one nucleus of spin quantum number $S \geq 1$. The nucleus of $S \geq 1$ placed in a surrounding that generates an electric field gradient (EFG) at its position exhibits a quadrupole interaction. The interaction Hamiltonian has the form: ${ }^{27-29}$

$$
H_{\mathrm{Q}}(S)(\Omega)=\frac{1}{2} \sqrt{\frac{3}{2}} \frac{a_{\mathrm{Q}}}{S(2 S-1)} \sum_{m=-2}^{2}(-1)^{m} V_{-m}^{2}(\Omega) T_{m}^{2}(S)
$$

where the quadrupole coupling constant, $a_{Q}$, is defined as $a_{Q}=e^{2} q Q / \hbar ; Q$ denotes the quadrupole moment of the nucleus, while $q$ is the $z z$ component of the EFG tensor at the position of the nucleus. The tensor components are defined as: $T_{0}^{2}(S)=\frac{1}{\sqrt{6}}\left[3 S_{z}^{2}-S(S+1)\right]$, $T_{ \pm 1}^{2}(S)=\mp \frac{1}{2}\left[S_{z} S_{ \pm}+S_{ \pm} S_{z}\right]$ and $T_{ \pm 2}^{2}(S)=\frac{1}{2} S_{ \pm} S_{ \pm}$. The Hamiltonian depends on an angle $\Omega$ describing the orientation of the principal axes system of the EFG tensor with respect to a reference frame. The quantities $V_{m}^{2}(\Omega)$ are defined as: $V_{m}^{2}(\Omega)=D_{0, m}^{2}(\Omega)+\frac{\eta}{\sqrt{6}}\left(D_{-2, m}^{2}(\Omega)+D_{2, m}^{2}(\Omega)\right)$ , where $\eta$ denotes the asymmetry parameter of the quadrupole interaction, while $D_{n, m}^{2}(\Omega)$ are Wigner rotation matrices of the $\Omega$ angle. 
When a nucleus possessing a quadrupole moment is placed in an external magnetic field, its energy level structure is determined by a superposition of the Zeeman and quadrupole interactions. The total Hamiltonian describing the energy levels is given as: $H_{0}(S)(\Omega)=H_{Z}(S)+H_{Q}(S)(\Omega)$, where $H_{Z}(S)=\omega_{S} S_{z} \quad\left(\omega_{S}=\gamma_{S} B_{0}\right.$, where $\gamma_{S}$ denotes the gyromagnetic factor of the $S$ spin nucleus), the angle $\Omega$ describes the orientation of the principal axes system of the EFG tensor with respect to the direction of the external magnetic field. At the same time a nucleus of spin quantum number $I=1 / 2$ (let us focus on ${ }^{1} \mathrm{H}$ ) can occupy two states described by the magnetic quantum numbers $m_{I}= \pm 1 / 2$ as a result of its Zeeman interaction: $H_{Z}(I)=\omega_{H} S_{z}\left(\omega_{H}=\gamma_{H} B_{0}\right.$, where $\gamma_{H}$ denotes the ${ }^{1} \mathrm{H}$ gyromagnetic factor). Thus, there are magnetic fields at which the ${ }^{1} \mathrm{H}(I=1 / 2)$ energy level splitting matches one of the transitions of the quadrupole nucleus between its energy levels. ${ }^{14-25,31-33}$ The ${ }^{1} \mathrm{H}$ and the quadrupole nucleus are mutually coupled by a magnetic dipole-dipole interaction. Dynamics in solids is often anisotropic that leads to a non-zero long time average of the dipoledipole coupling, referred to as a residual dipolar interaction. In consequence, the dipole-dipole coupling, $\quad H_{D D}(I, S)(t)$, can be split into two parts: $H_{D D}(I, S)(t)=\left\langle H_{D D}(I, S)\right\rangle+\left(H_{D D}(I, S)(t)-\left\langle H_{D D}(I, S)\right\rangle\right)$, where $\left\langle H_{D D}(I, S)\right\rangle=H_{D D}^{r e s}(I, S)$ is the residual dipolar coupling, while $\left(H_{D D}(I, S)(t)-\left\langle H_{D D}(I, S)\right\rangle\right)=H_{D D}^{\prime}(I, S)(t)$ denotes deviations of the total Hamiltonian from its averaged value (a fluctuating part of the dipole-dipole coupling). The residual dipolar coupling provides a pathway for polarization (magnetization) transfer between the participating nuclei. When the ${ }^{1} \mathrm{H}$ transition frequency matches one of the $S$ spin frequencies, the ${ }^{1} \mathrm{H}$ magnetization can be taken over by $S$ spin nucleus that manifests itself as a frequency specific decay of the ${ }^{1} \mathrm{H}$ magnetization. This effect is referred to as polarization transfer ${ }^{30-34}$ and can be quantitatively described by the time -independent Liouville equation. The fluctuating part of the dipole-dipole interaction causes ${ }^{1} \mathrm{H}$ spin-lattice relaxation. The relaxation also becomes faster at the magnetic fields at which the transition frequencies match. The ${ }^{1} \mathrm{H}$ and $S$ spin transitions are coupled and when the frequencies match the $S$ spin transitions enhance the efficiency of the ${ }^{1} \mathrm{H}$ transitions and hence the ${ }^{1} \mathrm{H}$ spin-lattice relaxation. ${ }^{20,21,33,34}$ This effect is referred to as QRE. 


\section{QUADRUPOLE RELAXATION ENHANCEMENT IN LIQUIDS}

The critical point is that to think about exploiting the QRE effect in MRI one must be able to observe a frequency specific ${ }^{1} \mathrm{H}$ spin-lattice relaxation enhancement in solution of ${ }^{209} \mathrm{Bi}$ containing species, for the solvent protons - in analogy to paramagnetic contrast agents. In solution the ${ }^{209} \mathrm{Bi}$ containing species undergoes rotational dynamics that modulates the orientation of the EFG tensor with respect to the external magnetic field. When the dynamics is very fast the quadrupole interaction gets averaged out from the perspective of the reference frame determined by the direction of the magnetic field and, in consequence, it acts as a relaxation mechanism for the quadrupole nucleus. In such a case quadrupole peaks do not exist - the ${ }^{1} \mathrm{H}$ relaxation is affected by the quadrupole spin relaxation acting as an additional source of modulations of the ${ }^{1} \mathrm{H}-{ }^{209} \mathrm{Bi}$ dipole-dipole coupling. When the rotational motion occurs on an intermediate time scale, the quadrupole interaction neither contributes to the energy level structure (creating the already described picture of QRE in solids) nor acts as a relaxation mechanism. In such a case the influence of the quadrupole spin dynamics on the ${ }^{1} \mathrm{H}$ relaxation cannot be any more just directly related to the concept of matching transition frequencies. A quantitative description of the ${ }^{1} \mathrm{H}$ spin-lattice relaxation for arbitrary motional conditions has been provided in Ref. ${ }^{20,21}$ The approach is based on the stochastic Liouville equation. The theory has been outlined in Appendix A, on the basis of Ref. 1,2,8-13,19-21,35-38

Let us quantitatively compare the PRE and QRE effects. When the motion responsible for the modulations of the inter-spin dipolar coupling (for instance rotation of the molecule carrying both spins) is fast (much faster than the relaxation itself), the corresponding ${ }^{1} \mathrm{H}$ spin-lattice relaxation rates originating from the $I-S$ dipole - dipole coupling are given as: ${ }^{1,2,39-41}$

$$
\begin{aligned}
R_{1}^{P R E}\left(\omega_{H}\right) & =\frac{2}{15}\left(\frac{\mu_{0}}{4 \pi} \frac{\gamma_{H} \gamma_{e} \hbar}{r^{3}}\right)^{2} S(S+1)\left[J\left(\omega_{\mathrm{e}}-\omega_{H}\right)+3 J\left(\omega_{H}\right)+6 J\left(\omega_{H}+\omega_{\mathrm{e}}\right)\right] \\
& \cong \frac{2}{15}\left(\frac{\mu_{0}}{4 \pi} \frac{\gamma_{H} \gamma_{e} \hbar}{r^{3}}\right)^{2} S(S+1)\left[7 J\left(\omega_{\mathrm{e}}\right)+3 J\left(\omega_{H}\right)\right] \\
R_{1}^{Q R E}\left(\omega_{H}\right) & =\frac{2}{15}\left(\frac{\mu_{0}}{4 \pi} \frac{\gamma_{H} \gamma_{S} \hbar}{r^{3}}\right)^{2} S(S+1)\left[J\left(\omega_{H}-\omega_{S}\right)+3 J\left(\omega_{H}\right)+6 J\left(\omega_{H}+\omega_{\mathrm{S}}\right)\right]
\end{aligned}
$$

where $R_{1}^{P R E}\left(\omega_{H}\right)$ and $R_{1}^{Q R E}\left(\omega_{H}\right)$ denote the ${ }^{1} \mathrm{H}$ spin-lattice relaxation rates for PRE and QRE, respectively; $\omega_{H}$ denotes the ${ }^{1} \mathrm{H}$ resonance frequency, $\omega_{e}=656 \omega_{H}$ is the electron resonance frequency, while $\omega_{S}$ denotes the resonance frequency of the quadrupole nucleus (as already explained). As $\omega_{e}>>\omega_{H}$ in Eq. (2) the approximation: $\omega_{e} \pm \omega_{H} \cong \omega_{e}$ has been used. The other 
symbols have the following meaning: $\gamma_{e}$ and $\gamma_{S}$ denote the electron and the quadrupole nucleus gyromagnetic factors, respectively, $r$ is the inter-spin distance. The $J(\omega)$ quantities are referred to as spectral density functions; for the case of rotational dynamics they can be expressed as: $:^{1,2,8-}$ 11,39

$$
J(\omega)=\frac{\tau_{\text {eff }}}{1+\left(\omega \tau_{\text {eff }}\right)^{2}}, \quad \tau_{e f f}^{-1}=\tau_{r o t}^{-1}+R_{e(Q)}+\tau_{e x}^{-1}
$$

where $\tau_{r o l}$ and $\tau_{e x}$ denote the rotational correlation time and the exchange life time, respectively, while $R_{e(Q)}$ is electronic (quadrupole) relaxation. Taking ${ }^{209} \mathrm{Bi}(S=9 / 2)$ as an example of a quadrupole nucleus and neglecting the electronic (quadrupole) relaxation one can calculate the ratio $\frac{R_{1, H}^{P R E}}{R_{1, H}^{Q R E}}=\frac{7}{11}\left(\frac{\gamma_{e}}{\gamma_{S}}\right)^{2}=1.35^{*} 10^{7}$ (assuming the same inter-spin distance) in the low frequency (magnetic field) limit (the spin quantum number $S=7 / 2$ for $\mathrm{Gd}^{3+}$ has been taken into account). At high magnetic fields the ratio can get smaller by factor $3 / 7$ due to the spectral density $J\left(\omega_{e}\right)$ vanishing when the range $\omega_{e} \tau_{\text {rot }}>>1$ is reached. Thus, for fast dynamics the use of QRE as a contrast mechanism is out of question, in contrary to PRE effects which always ensure ${ }^{1} \mathrm{H}$ spin- lattice relaxation enhancement due to the strong proton - electron dipole - dipole coupling.

The paramagnetic centre can be immobilized, for instance by binding to large protein molecules that ensures slow tumbling in water. One distinguishes between inner-sphere and outer-sphere PRE. Inner - sphere PRE is associated with water molecules forming a coordination sphere around the species carrying the paramagnetic centre and rotating with it as a whole body.,2,811,15 There are three factors modulating the proton spin - electron spin dipole-dipole coupling as seen in Eq. (4). The first one is the rotational dynamics, but it can be, in fact, neglected as it is purposely slow. The next factor is the electron spin relaxation which is caused by fluctuations of Zero Field Splitting (ZFS) interaction around its averaged (residual) value. The fluctuations are caused by internal dynamics of the molecule containing the lanthanide ions. The third factor is the exchange lifetime of water molecules between the coordination sphere and bulk water. The electron spin relaxation is very complex. The main reason for that is the energy level structure of the electron spin. The energy level structure is determined by a superposition of the electron spin Zeeman coupling and the ZFS interactions. For slow molecular tumbling one can assume that the principal axis system of the ZFS interaction is fixed with respect to the direction of the external magnetic field leading to an orientation dependent energy level structure (the 
overall PRE effect is a result of averaging over all molecular orientations). This complex energy level structure combined with the high spin quantum number leads to multi-exponential electron spin relaxation characterised by several, field dependent relaxation rates. The electron spin relaxation influences the ${ }^{1} \mathrm{H}$ relaxation in two ways. First, as it is fast (due to strong ZFS) it very considerably influences (reduces in the low and intermediate frequency range) the ${ }^{1} \mathrm{H}$ spin-lattice relaxation rate. The ${ }^{1} \mathrm{H}$ relaxation rate is additionally reduced by the ZFS contribution to the electron spin energy level structure. In consequence, for $1 \mathrm{mM}$ concentration of the paramagnetic molecules the enhanced relaxation rate typically yields a value being by about 10-50 times higher ${ }^{3,14,15,42,43}$ compared to the water ${ }^{1} \mathrm{H}$ spin-lattice relaxation rate. In the case of very slow molecular tumbling enhancement values up to 200 can be reached. ${ }^{44-46}$

The second effect associated with the electron spin relaxation is that the presence of multiple frequency dependent relaxation rates can lead to a single, rather broad maximum of the ${ }^{1} \mathrm{H}$ spinlattice relaxation rate on the top of the overall PRE. The experimentally obtained ${ }^{1} \mathrm{H}$ spin-lattice relaxation rate $\widetilde{R}_{1}^{P R A}\left(\omega_{H}\right)$ yields (after extracting the relaxation contribution from pure water): $1,2,8-11,39,47,48$

$\widetilde{R}_{1}^{P R E}\left(\omega_{H}\right)=\frac{P q}{\left(R_{1}^{P R E}\left(\omega_{H}\right)\right)^{-1}+\tau_{e x}}$

where $q$ is the coordination number (number of water molecules in the coordination sphere of a single paramagnetic centre), while $P=c / 55.6$, where $c$ denotes the concentration of the paramagnetic centres. An analogous expression can be set up for $\widetilde{R}_{1}^{Q R A}\left(\omega_{H}\right)$ :

$\widetilde{R}_{1}^{Q R E}\left(\omega_{H}\right)=\frac{P q}{\left(R_{1}^{Q R E}\left(\omega_{H}\right)\right)^{-1}+\tau_{e x}}$

In this case $q$ is the number of water molecules in the coordination sphere of a single ${ }^{209} \mathrm{Bi}$ centre), $P=c / 55.6$, where $c$ denotes the concentration of the quadrupole particles in mole, multiplied by the number of ${ }^{209} \mathrm{Bi}$ nuclei per particle, $n$.

One should clearly stress at this point that neither Eq. (2) nor Eq. (3) is valid outside the limit of fast motion. The description of $R_{1}^{P R E}\left(\omega_{H}\right)$ and $R_{1}^{Q R E}\left(\omega_{H}\right)$ is then much more cumbersome and it is based on the Stochastic Liouville Equation (SLE) as pointed out in Section II.

Eqs. (5a) show the double role of the exchange dynamics; it contributes to the fluctuations of the proton spin - electron spin dipole-dipole coupling (and is included in the approach in terms of the SLE) and also appears in the denominator of Eqs. (5a). The situation is simpler for QRE 
as $R_{1}^{Q R E}\left(\omega_{H}\right)$ is much smaller than $R_{1}^{P R E}\left(\omega_{H}\right)$ and, hence one can be sure that the condition: $\left(R_{1}^{Q R E}\left(\omega_{H}\right)\right)^{-1}>\tau_{e x}$ holds. Thus, Eq. (5b) for QRE simplifies to the form:

$\widetilde{R}_{1}^{Q R E}\left(\omega_{H}\right)=P q R_{1}^{Q R E}\left(\omega_{H}\right)$

This means that in the case of QRE the exchange motion is only important as a source of modulations of the $I-S$ dipole - dipole interaction.

A possible way of slowing down the rotational dynamics is embedding the molecules carrying the quadrupole nucleus into a nanoparticle. The pertinent question to be asked in this context is whether the QRE can be large enough to be useful for practical applications. The strong quadrupole interactions of 209Bi that leads to quadrupole peaks at magnetic fields close to $3 \mathrm{~T}$ (this is a typical magnetic field of MRI scanners) have experimentally been confirmed for solid tris(2-methoxyphenyl)bismuthane for which the amplitude of the quadrupole coupling yields: $a_{Q}=715.27 \mathrm{MHz}$, while the asymmetry parameter is $\eta=0 .^{25,36}$ Fig. 1 shows simulations of the $R_{1}^{Q R E}\left(\omega_{H}\right)$ values versus ${ }^{1} \mathrm{H}$ resonance frequency for these parameters and different values of the rotational correlation time, $\tau_{\text {rot }}$. The simulations have been performed in terms of a general theory of QRE ${ }^{20,21}$ outlined in Appendix A. The quadrupole relaxation has been neglected. This is a next difference between QRE and PRE - the electron spin relaxation is fast, while the quadrupole relaxation in many cases is slow enough to be omitted, at least for the purpose of the estimations discussed here. The inter-spin distance has been set to $r=2.0 \AA$. To achieve such a short distance, the ${ }^{209} \mathrm{Bi}$ nuclei have to be placed on the surface of the nanoparticles. Exchange dynamics has not been taken into account in the simulations (it has been assumed that it does not influence the fluctuations of the dipole-dipole coupling in a significant way). One can observe a progressive formation of the quadrupole peaks when the rotational correlation time varies from $\tau_{\text {rot }}=1 * 10^{-8} \mathrm{~s}$ to $\tau_{r o t}=1 * 10^{-6} \mathrm{~s}$. The lower limit corresponds to the case described by Eq. (3), while the higher limit corresponds to the case of immobilized systems. One should also be aware that the $I-S$ dipole-dipole coupling is modulated by the exchange dynamics and one should rather not expect the exchange lifetime, $\tau_{e x}$, being longer than $1 * 10^{-6} \mathrm{~s}^{49-53}$ The rotational correlation time can be linked to the radius, $R$, of the nanoparticle by the StokesEinstein equation. For water at room temperature $\tau_{r o t}=1 * 10^{-9} \mathrm{~s}$ corresponds to $R \cong 1 \mathrm{~nm}$, for $\tau_{\text {rot }}=5^{*} 10^{-7} \mathrm{~s}$ one gets $R \cong 8 \mathrm{~nm}$, while for $\tau_{r o t}=1 * 10^{-6} \mathrm{~s}$ one gets $R \cong 10 \mathrm{~nm}$. In Fig. 1 the level of water ${ }^{1} \mathrm{H}$ spin-lattice relaxation rate, $R_{1, \text { water }}\left(\omega_{H}\right) \cong 0.4 \mathrm{~s}^{-1},{ }^{47}$ has been indicated for 
comparison. The QRE peaks labelled as A, B, C, D correspond to specific ${ }^{209} \mathrm{Bi}$ transitions between its eigenstates; this subject has been discussed in detail in Ref. ${ }^{25}$

Fig.2 shows the ratio $x=\frac{R_{1}^{Q R E}}{R_{1, \text { water }}}$ plotted for the quadrupole peaks labelled as A, B, C, D versus the nanoparticle radius, $\mathrm{R}$. The ratio is an outcome of a competition: a longer $\tau_{\text {rot }}$ means more pronounced quadrupole peaks, but at the same time at high frequencies the overall $\widetilde{R}_{1}^{Q R E}\left(\omega_{H}\right)$ value becomes lower. One can see from Fig.2 that the ratio $\mathrm{x}$ for peak D (corresponding to 3T scanners $)$ is of the order of about 4.5 when approaching the nanoparticle radius of $8 \mathrm{~nm}\left(\tau_{\text {rot }} \cong\right.$ $\left.5^{*} 10^{-7} \mathrm{~s}\right)$ and about 9 for the radius of $10 \mathrm{~nm}\left(\tau_{r o t} \cong 1 * 10^{-6} \mathrm{~s}\right)$. For peak B (corresponding to 1.5 T scanners) the ratios are about 57 for the radius of $8 \mathrm{~nm}$ and about 100 for the radius of $10 \mathrm{~nm}$. For peaks $\mathrm{A}$ and $\mathrm{C}$ the ratios are even higher. Yet, the crucial point is that one should reach such values not for the ratio $x=\frac{R_{1}^{Q R E}}{R_{1, \text { water }}}$, but for $\tilde{x}=\frac{\widetilde{R}_{1}^{Q R E}}{R_{1, \text { water }}}$, where $\widetilde{R}_{1}^{Q R E}=P q R_{1}^{Q R E}$. As we expect a lower enhancement in the case of QRE agents let us consider a factor 10 higher concentration (i.e. $10 \mathrm{mM}$ ). For this concentration one gets: $\widetilde{R}_{1}^{Q R E} \cong 2 * 10^{-4} q n R_{1}^{Q R E}$. One can assume that about 5 molecules carrying ${ }^{209} \mathrm{Bi}$ can be placed in the vicinity of the surface of a nanoparticle of the radius of $1 \mathrm{~nm}$. As the value scales with $R^{2}$ one gets for nanoparticles of 10 nm radius the value $n \cong 500$, so $\widetilde{R}_{1}^{Q R E} \cong 0.1 q R_{1}^{Q R E}$. Taking into account only the size of the nanoparticle and the size of water molecule one can expect the number of water molecules in the coordination sphere being of about $10^{4}$. As each ${ }^{209} \mathrm{Bi}$ nucleus effectively interacts only with water molecules in its vicinity one should consider here an effective $q$ value. Taking into account the ratio between the estimated numbers of water molecules in the coordination sphere and the number of ${ }^{209} \mathrm{Bi}$ on the nanoparticle surface, let us assume that $q$ of about 10 can be reached; this implies $\widetilde{R}_{1}^{Q R E} \cong R_{1}^{Q R E}$. One should, however, be aware that we assume here the most favourable conditions; the coordination number can be lower. For $q=10$ one obtains the $\tilde{x}$ ratio for peak $\mathrm{D}$ of the order of 4.5 for $R \cong 8 \mathrm{~nm}$ and in the order of 9 for $R \cong 10 \mathrm{~nm}$. Analogous values for peak B yield 57 and 100, respectively. This implies that for $1 \mathrm{mM}$ concentration one can expect relaxivity of the order of $(2.4-4) /\left(\mathrm{s}^{*} \mathrm{mM}\right)$ at about $1.5 \mathrm{~T}$. It is worth to note that the relaxivity of Gd-based products like Gadovist in blood plasma at $1.5 \mathrm{~T}$ is about $(4-7) /\left(\mathrm{s}^{*} \mathrm{mM}\right) .{ }^{42}$ Analogously, the Gd-based relaxivity at 3 T yields $(3-5.4) /\left(\mathrm{s}^{*} \mathrm{mM}\right),{ }^{43}$ while our estimation for QRE at peak D gives about $(0.2-0.35) /(\mathrm{s} * \mathrm{mM})$. 
At present we are far from reaching so large QRE values, however, we are able to demonstrate that QRE effects can indeed be observed for liquids. This confirmation encourages us to further pursuit the research in the direction of producing suitable nanoparticle systems.

To verify the concept of QRE being present for intermediate dynamics it is necessary to perform relaxation experiments for a solution, ideally in water (from the perspective of MRI). As the water solubility of $\mathrm{t}$ tris(2-methoxyphenyl)bismuthane is limited we have decided for tetrahydrofuran (THF) solution. The experimental details and results are presented in the next sections.

\section{EXPERIMENTAL DETAILS \\ IV.1 Details of NQR and NMR experiments}

In the first step Nuclear Quadrupole Spectroscopy (NQR) experiments have been performed for selected ${ }^{209} \mathrm{Bi}$ compounds to determine the quadrupole parameters $\left(a_{Q}\right.$ and $\left.\eta\right)$ from the positions of the NQR lines (diagonalizing the Hamiltonian of Eq. 1). The results have been presented in Ref. ${ }^{25,26}$. For tris(2-methoxyphenyl)bismuthane $\left(\mathrm{C}_{21} \mathrm{H}_{21} \mathrm{BiO}_{3}\right)$ at $310 \mathrm{~K}$ four NQR transitions have been detected at the frequencies of $29.87 \mathrm{MHz}, 59.64 \mathrm{MHz}, 89.47 \mathrm{MHz}$ and 119.27MHz. The frequencies correspond to the spin transitions $3 / 2 \rightarrow 1 / 2,5 / 2 \rightarrow 3 / 2,7 / 2 \rightarrow 5 / 2$ and $9 / 2 \rightarrow 7 / 2$, respectively, for $a_{Q}=715.3 \pm 0.4 \mathrm{MHz}$ and $\eta=0 \pm 0.001$ (the theoretical frequencies are $29.80 \mathrm{MHz}, 59.61 \mathrm{MHz}, 89.41 \mathrm{MHz}$ and $119.21 \mathrm{MHz}$, respectively). ${ }^{25,26}$

The NQR data have been collected by using two complementary pulse type NQR spectrometers; a home built one "Graz-NQRS-MK1'” which was used for localizing peaks with a wide band fast scan up to $50 \mathrm{MHz}$ and a "Scout' (Tecmag, Inc., USA) used for more accurate narrowband scans to resolve finer features. Both spectrometers are driven by a $500 \mathrm{~W}$ power amplifier that can supply a set of different solenoid RF-coils of $10 \mathrm{~mm}$ diameter all together covering a range of $20 \mathrm{MHz}$ up to $150 \mathrm{MHz}$.

In the next step ${ }^{1} \mathrm{H}$ NMR relaxation experiments versus frequency (magnetic field) have been performed by using HTC-110 3T superconducting magnet equipped with standalone PC-NMR console (Stelar) for ${ }^{1} \mathrm{H}$ Larmor frequency range $20 \mathrm{MHz}-128 \mathrm{MHz}$ and at temperatures between $293 \mathrm{~K}-185 \mathrm{~K}$. Standard inversion recovery sequence $\left(180^{\circ}-\tau-90^{\circ}\right)$ was used with $90^{\circ}$ pulse length between 3-5 $\mu$ s depending on the frequency. The sample temperature has been controlled by built-in VTC unit and an independent thermocouple with the stability of $0.2 \mathrm{~K}$. ${ }^{1} \mathrm{H}$ spinlattice relaxation data have been collected for pure tetrahydrofuran (THF) and THF solution of 
tris(2-methoxyphenyl)bismuthane $(50 \mathrm{mM})$. In all cases the relaxation has turned out to be single exponential. Examples of ${ }^{1}$ Hmagnetization curves recorded versus time are shown in Appendix B, that also includes the relaxation data for pure THF.

\section{IV.2. Sample preparation}

As synthetic concept, Grignard type reaction starting from $\mathrm{BiCl}_{3}$ and the $\mathrm{ArMgBr}$ was employed to synthesise tris(2-methoxyphenyl)bismuthane. After completion of the reaction the inorganic salts are filtered off and the pale yellow solutions are subjected to an aqueous standard workup procedure. Crystallisation from toluene at $-35^{\circ}$ yielded colourless crystals of the tris $(2-$ methoxyphenyl)bismuthane in good yield. A stable tetrahydrofuran (THF) solution $(\sim 50 \mathrm{mM})$ was prepared by dissolving $15.5 \mathrm{mg}$ of tris(2-methoxyphenyl)bismuthane powder in $0.6 \mathrm{~mL}$ of THF (anhydrous, \%99.9 from Sigma-Aldrich) at room temperature under stirring. The solution was then transferred to standard $5 \mathrm{~mm}$ glass NMR tube and then glass was sealed after evacuating the air in the tube.

\section{RESULTS AND ANALYSIS}

The results of ${ }^{1} \mathrm{H}$ spin-lattice relaxation experiments for THF solution of tris(2methoxyphenyl)bismuthane are shown in Fig.3. The concentration of tris(2methoxyphenyl)bismuthane $(50 \mathrm{mM})$ gives the ratio between the protons of the solute and solvent molecules of about 0.01 ; this ensures that in the experiment essentially the signal of solvent protons has been detected. Although the QRE effect is relatively weak (as expected for fast dynamics), it is present at all temperatures. In such a case it is of primary importance to investigate the experimental errors. The error bars are not present in Fig. 3 as they do not exceed the size of the symbols (in terms of numbers they do not exceed 1\%). Appendix B includes ${ }^{1} \mathrm{H}$ spin-lattice relaxation data for pure THF collected at the corresponding temperatures.

The QRE effect is most pronounced at the highest temperature $(295 \mathrm{~K})$ at which the rotational dynamics is most fast. Indeed, considering only the rotational dynamics, the effect should be opposite, but one has to be aware of the possible role of other factors (temperature and frequency dependent multi-exponential ${ }^{209} \mathrm{Bi}$ relaxation and temperature dependent exchange dynamics). From the simulations presented in Fig. 2 one can conclude that to see the QRE effects the rotational correlation time should not be shorter than $(2-5)^{*} 10^{-8} \mathrm{~s}$. Knowing the solvent viscosity, $\eta$, one can estimate from the Stokes-Einstein equation $\left(\tau_{\text {rot }}=\frac{4}{3} \frac{\pi \eta r^{3}}{k_{B} T}\right)$ the molecular radius, $r$, for which the requested correlation time can be achieved. For THF such 
a value of $\tau_{\text {rot }}$ can be obtained for the molecular size of about $3.4 \mathrm{~nm}-4.7 \mathrm{~nm}$. This indicates that the THF solution of tris(2-methoxyphenyl)bismuthane contains, in fact, clusters of the ${ }^{209} \mathrm{Bi}$ containing molecules that in this case works to our advantage.

\section{CONCLUSIONS}

Aiming at exploring the potential of the concept of the QRE based contrast mechanism for MRI, a thorough analysis of the achievable QRE has been presented. It has been concluded that the concept of QRE-based contrast agents including nanoparticles as carriers of ${ }^{209} \mathrm{Bi}$ nuclei is promising. One should be able to obtain sufficiently large QRE effects provided the following conditions are fulfilled:

- ${ }^{209} \mathrm{Bi}$ nuclei are placed on the surface of the nanoparticles to ensure a short distance to the solvent (water) protons. When talking about 'surface' one is not restricted to the outer shape of the particle. The effective surface could, e.g., be significantly enlarged by using "sponge-like" structures.

- The nanoparticles have appropriate size optimizing the competition between the amplitude of the quadrupole peaks (that increase with the increasing rotational correlation time) and the amplitude of the spectral density (that can at high magnetic fields considerably decrease for longer correlation times). Here one should also take into account clinical limitations of the nanoparticle sizes.

- The distribution of the nanoparticle sizes is narrow. Imagine that the ${ }^{1} \mathrm{H}-{ }^{209} \mathrm{Bi}$ relaxation contribution is a sum of the curves shown in this figure taken with different weight factors (according to the distribution of the sizes) - the resulting curve can likely show relatively week QRE effects.

- The quadrupole relaxation is long (longer than the rotational correlation time and the exchange life time) so that it does not contribute to the fluctuations of the ${ }^{1} \mathrm{H}_{-}{ }^{209} \mathrm{Bi}$ dipoledipole coupling.

- The exchange life time is long (preferably $10^{-7} \mathrm{~s}-10^{-6} \mathrm{~s}$ ).

To confirm the possibility to obtain QRE in liquids ${ }^{1} \mathrm{H}$ spin-lattice experiments have been performed for THF solution of tris(2-methoxyphenyl)bismuthane. The experiments have revealed QRE peaks in the liquid state as predicted by a general (based on the Stochastic Liouville equation) relaxation theory of $1 / 2$ - spin relaxation caused by dipole-dipole interactions with quadrupole nuclei. The transfer of the QRE effects from solid samples to liquid samples is an important prerequisite for the usage as an MRI contrast agent. The 
presented results are the first example of QRE effects observed in solution. They give a strong indication that novel MRI contrast agents based on QRE with molecules containing high-spin nuclei are feasible.

Pursuing this idea and profiting from results obtained so far, we shall investigate, in the next step, QRE effects for solutions of tris(2-methoxyphenyl)bismuthane containing nanoparticles of various sizes.

\section{ACKNOWLEDGEMENT}

This project has received funding from the European Union's Horizon 2020 Research and Innovation Programme under grant agreement No 665172. 


\section{REFERENCES}

1. J. Kowalewski, D. Kruk, and G. Parigi, Adv. Inorg. Chem. 57, 41 (2005).

2. E. Belorizky, P. H. Fries, L. Helm, J. Kowalewski, D. Kruk, R. R. Sharp, and P.-O. Westlund, J. Chem. Phys. 128, 052315 (2008).

3. P. Caravan, Chem. Soc. Rev. 35, 512 (2006).

4. A. S. Merbach, L. Helm, and É. Tóth, The Chemistry of Contrast Agents in Medical Magnetic Resonance Imaging (Wiley \& Sons. Ltd., Chichester, 2013), p. 1-471.

5. S. Aime, M. Botta, and E. Terreno, Adv. Inorg. Chem. 57, 173 (2005).

6. S. Laurent, C. Henoumont, L. Vander Elst, and R. N. Muller, Eur. J. Inorg. Chem. 2012, 1889 (2012).

7. A. Datta and K. N. Raymond, Acc. Chem. Res. 42, 938 (2009).

8. I. Bertini, O. Galas, C. Luchinat, and G. Parigi, J. Magn. Reson. Series A 113, 151 (1995).

9. T. Nilsson, J. Svoboda, P.-O. Westlund, and J. Kowalewski, J. Chem. Phys. 109, 6364 (1998).

10. D. Kruk, T. Nilsson, and J. Kowalewski, Phys. Chem. Chem. Phys. 3, 4907 (2001).

11. D. Kruk and J. Kowalewski, Mol. Phys. 101, 2861 (2003).

12. P.-O. Westlund, J. Chem. Phys. 108, 4945 (1998).

13. I. Bertini, J. Kowalewski, C. Luchinat, T. Nilsson, and G. Parigi, J. Chem. Phys. 111, 5795 (1999).

14. P. Caravan, J. J. Ellison, T. J. McMurry, and R. B. Lauffer, Chem. Rev. 99, 2293 (1999).

15. É. Tóth, L. Helm, and A. E. Merbach, in Contrast Agents I, edited by W. Krause (Springer-Verlag, Berlin, 2002) p. 61.

16. F. Winter and R. Kimmich, Mol. Phys. 45, 33 (1982).

17. F. Winter and R. Kimmich, Biophys. J. 48, 331 (1985).

18. D. J. Lurie, G. R. Davies, M. A. Foster, and J. M. Hutchison, Magn. Reson. Imaging 23, 175 (2005).

19. E. P. Sunde and B. Halle, J. Magn. Reson. 203, 257 (2010).

20. P.-O. Westlund, Phys. Chem. Chem. Phys. 12, 3136 (2010).

21. D. Kruk, A. Kubica, W. Masierak, A. F. Privalov, M. Wojciechowski, and W. Medycki, Solid State Nucl. Magn. Reson. 40, 114 (2011).

22. W. Masierak, M. Florek-Wojciechowska, I. Oglodek, R. Jakubas, A. F. Privalov, B. Kresse, F. Fujara, and D. Kruk, J. Chem. Phys. 142, 204503 (2015). 
23. M. Florek-Wojciechowska, M. Wojciechowski, R. Jakubas, S. Brym, and D. Kruk, J. Chem. Phys. 144, 054501-1 (2016).

24. M. Florek-Wojciechowska, R. Jakubas, and D. Kruk, Phys. Chem. Chem. Phys. 19, 11197 (2017).

25. D. Kruk, E. Umut, E. Masiewicz, C. Sampl, R. Fischer, S. Spirk, C. Gösweiner, and H. Scharfetter, Phys. Chem. Chem. Phys. 20, 12710 (2018).

26. C. Gösweiner, P. Lantto, R. Fischer, C. Sampl, E. Umut, P-O. Westlund, D. Kruk, M. Bödenler, S. Spirk, A. Petrovič, and H. Scharfetter, Phys. Rev. X 8, 021076 (2018).

27. C. Slichter, Principles of magnetic resonance (Springer-Verlag, Berlin, 1990).

28. R. Kimmich, NMR: Tomography, Diffusometry, Relaxometry (Springer-Verlag, Berlin, 2012).

29. D. Kruk, Understanding Spin Dynamics (Pan Stanford Publishing Pte Ltd, Singapore 2016).

30. M. Nolte, A. Privalov, J. Altmann, V. Anferov, and F. Fujara, J. Phys. D: Appl. Phys. 35, 939 (2002).

31. D. Kruk, J. Altmann, F. Fujara, A. Gädke, M. Nolte, and A. F. Privalov, J. Phys.: Condens Matter. 17, 519 (2005).

32. D. Kruk, F. Fujara, P. Gumann, W. Medycki, A. F. Privalov, and Ch. Tacke, Solid State Nucl. Magn. Reson. 35, 152 (2009).

33. D. Kruk, A. Privalov, W. Medycki, C. Uniszkiewicz, W. Masierak, and R. Jakubas, Annual Reports on NMR Spectroscopy 76, 67 (2012).

34. F. Fujara, D. Kruk, and A.F. Privalov, Prog. Nucl. Magn. Reson. Spectrosc. 82, 39 (2014).

35. T. Nilsson and J. Kowalewski, J. Magn. Reson. 146, 345 (2000).

36. P.-O. Westlund, in Dynamics of Solutions and Fluid Mixtures by NMR, edited J. J. Delpuech (Wiley \& Sons. Ltd., Chichester, 1995).

37. R. M. Lynden-Bell, Mol. Phys. 22, 837 (1971).

38. N. Benetis, J. Kowalewski, L. Nordenskiöld, H. Wennerström, and P.-O. Westlund, Mol. Phys. 48, 329 (1983).

39. I. Bertini, C. Luchinat, and G. Parigi, Solution NMR of Paramagnetic Molecules (Elsevier, Amsterdam, 2001).

40. A. Abragam, The Principles of Nuclear Magnetism (Oxford University Press, Oxford, 1961). 
41. J. Kowalewski and L. Mäler, Nuclear Spin Relaxation in Liquids: Theory, Experiments, and Applications (Taylor \& Francis, NewYork, 2006).

42. M. Rohrer, H. Bauer, J. Mintorovitch, M. Requardt, and H. J. Weinmann, Invest Radiol 40, 715 (2005).

43. Y. Shen, F. L. Goerner, C. Snyder, J. N. Morelli, D. Hao, D. Hu, X. Li, and V. M. Runge, Invest Radiol. 50, 330 (2015).

44. T.D.C. Viale, and S. Aime, Chem. Rev. 110, 3019 (2010).

45. A.D. Sherry and Y.Wu, Current Opinion in Chemical Biology 17, 167 (2013).

46. P. Hermann, J. Kotek, V. Kubíček and I. Lukeš, Dalton Trans. 23, 3027 (2008).

47. D. Kruk, E. Umut, E. Masiewicz, P. Hermann, and H. Scharfetter, Mol. Phys. DOI: 10.1080/00268976.2018.1517907 (2018).

48. M. Rubinstein, A. Baram, and Z. Luz, Mol. Phys. 20, 67 (1970).

49. K. M. Donahue, R. M. Weisskoff, and D. Burstein, J. Magn. Reson. Imaging. 7, 102 (1997).

50. R. M. Judd, S. B. Reeder, and K. May-Newman, Magn. Reson. Med. 41, 334 (1999).

51. A. D. Sherry and Y. Wu, Curr Opin Chem Biol. 17, 167 (2013).

52. B. N. Siriwardena-Mahanama and M. J. Allen, Molecules. 18, 9352 (2013).

53. P. Caravan, G. Parigi, J. M. Chasse, N. J. Cloutier, J. J. Ellison, R. B. Lauffer, C. Luchinat, S. A. McDermid, M. Spiller, and T. J. McMurry, Inorg. Chem. 46, 6632 (2007). 


\section{FIGURES}

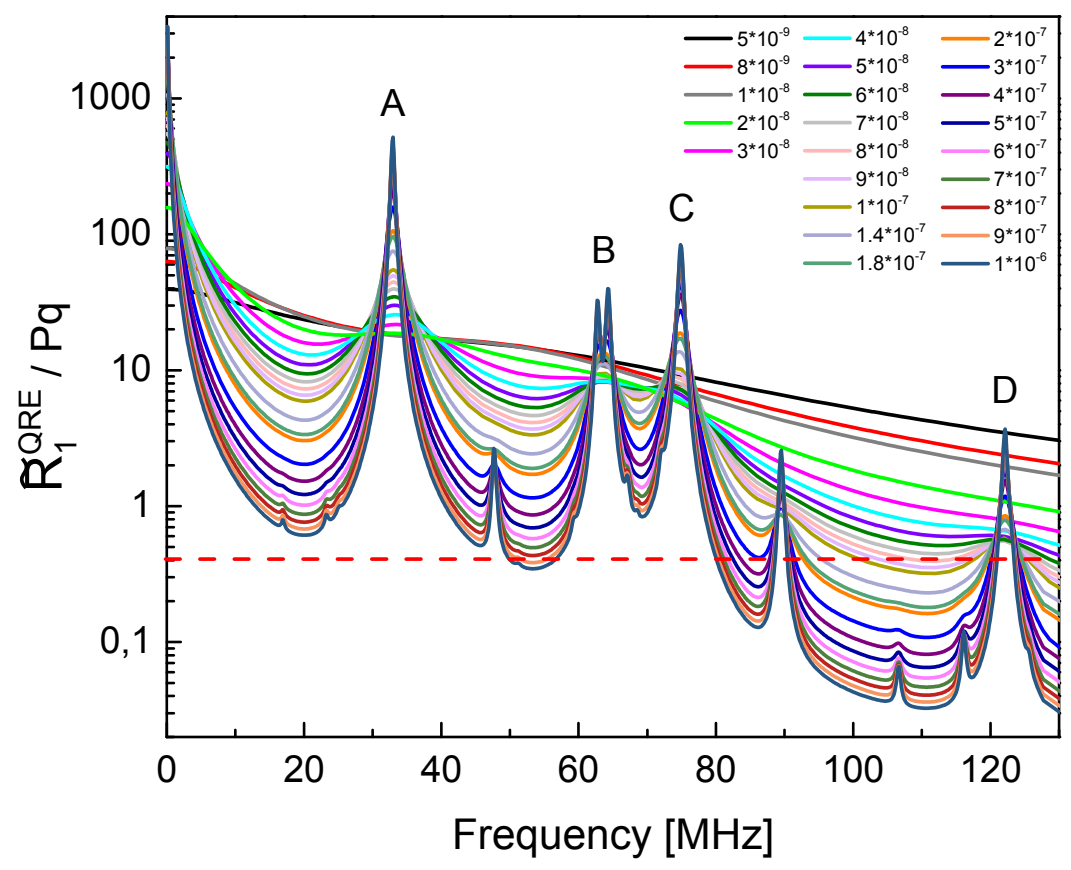

FIG. 1. Simulated $\frac{\widetilde{R}_{1}^{Q R E}}{P q}$ values for ${ }^{209} \mathrm{Bi}\left(\mathrm{a}_{\mathrm{Q}}=715.2 \mathrm{MHz}, \eta=0, r=2.0 \AA\right)$ depending on the rotational correlation time, $\tau_{\text {rot }}$. Dashed line indicates the ${ }^{1} \mathrm{H}$ spin-lattice relaxation rate. 


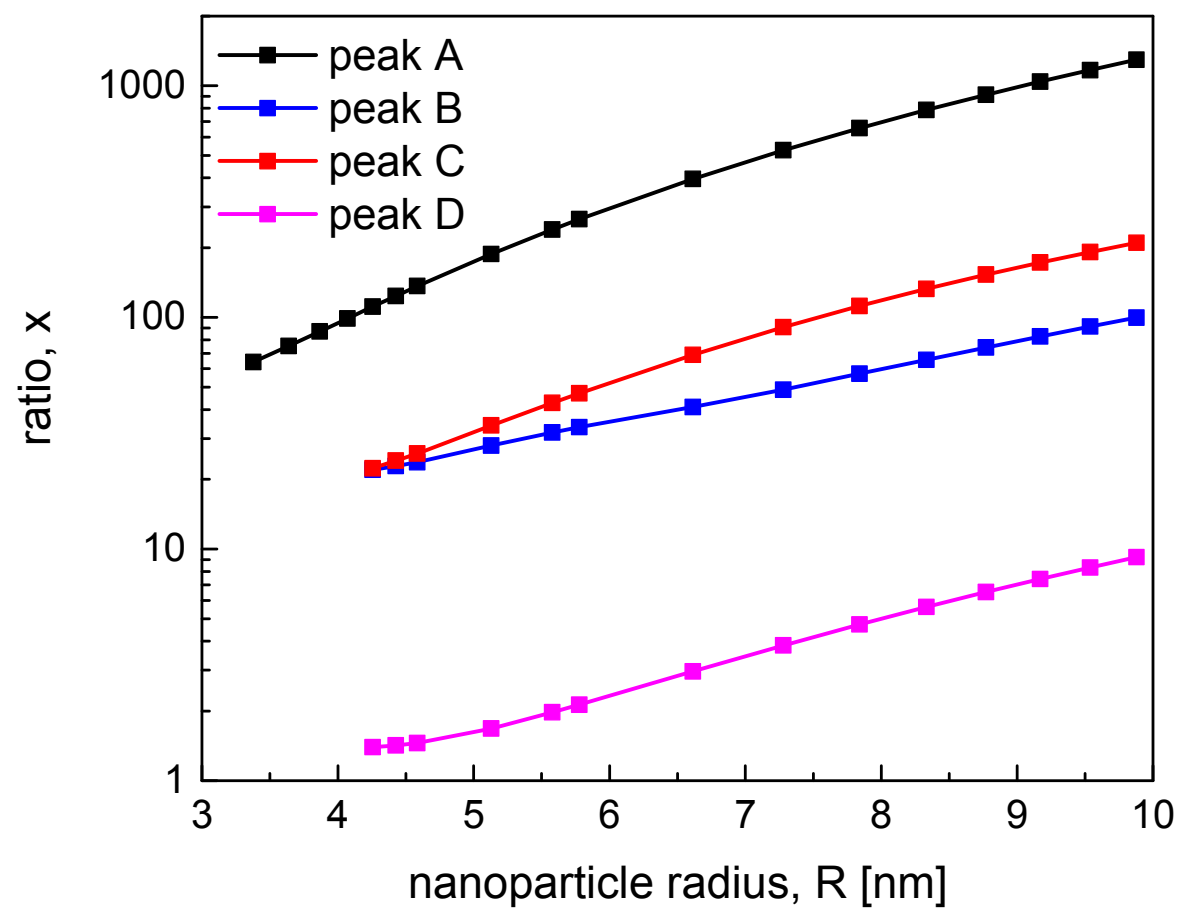

FIG. 2. $x=\frac{R_{1}^{Q R E}}{R_{1, \text { water }}}$ for the quadrupole peaks labelled as $\mathrm{A}, \mathrm{B}, \mathrm{C}, \mathrm{D}$ versus the nanoparticle radius, $R$ for water at room temperature; $R_{1}^{Q R E}$ taken from Fig. 1. 


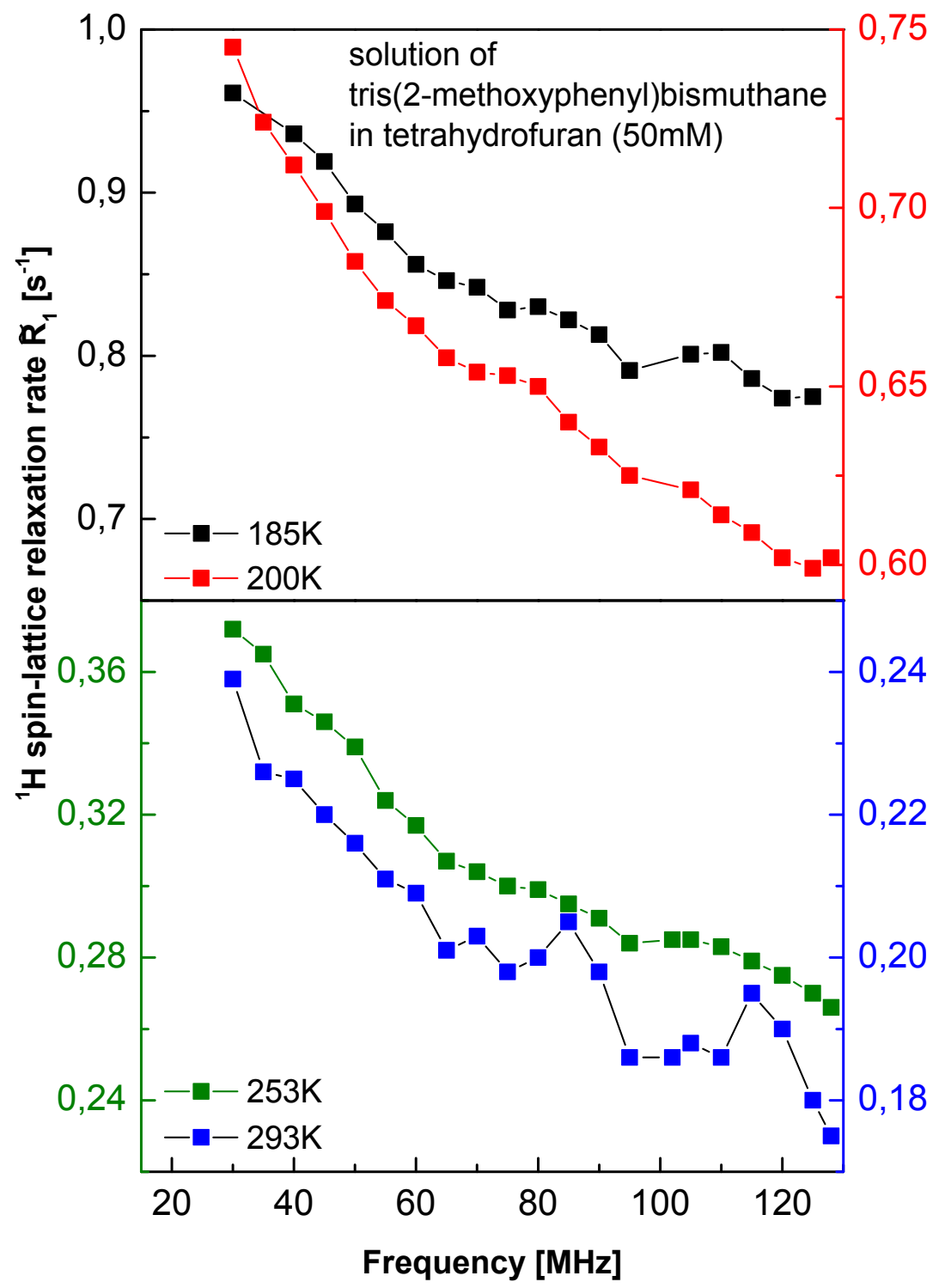

FIG. 3. ${ }^{1} \mathrm{H}$ spin-lattice relaxation rates, $\widetilde{R}_{1}$, versus frequency for THF solution of tris(2methoxyphenyl)bismuthane. 


\section{Appendix A}

\section{General theory of QRE - outline}

The $I-S$ dipole - dipole Hamiltonian as:

$$
H_{D D}(I, S, \Omega)=\sum_{m=-1}^{1}(-1)^{m} T_{m}^{1}(I) T_{-m}^{1, D D}(S, \Omega)
$$

where the rank one $I$ spin tensor operators are defined as: $T_{0}^{1}(I)=I_{z}, T_{ \pm}^{1}(I)=\mp I_{ \pm} / \sqrt{2}$, while the $T_{m}^{1, D D}(S, \Omega)$ tensors include the $S$ spin variables and the spacial degrees of freedom according to the definition: ${ }^{1,2,8-13,19-21,35-38}$

$$
T_{m}^{1, D D}(S, \Omega)=\sqrt{6}\left(\frac{\mu_{0}}{4 \pi} \frac{\gamma_{I} \gamma_{S} \hbar}{r^{3}}\right) \sqrt{\frac{5(2 S+1)(S+1) S}{3}} \sum_{q=-1}^{1}\left(\begin{array}{ccc}
2 & 1 & 1 \\
1-q & q & -1
\end{array}\right) S_{q}^{1} D_{0,1-q}^{2}(\Omega)
$$

The operator $S_{q}^{1}$ is defined as: $S_{q}^{1}=\sqrt{\frac{3}{(2 S+1)(S+1) S}} T_{q}^{1}(S)\left(T_{0}^{1}(S)=S_{z}, T_{ \pm 1}^{1}(S)=\mp S_{ \pm} / \sqrt{2}\right.$ ), while the Wigner rotation matrices $D_{0,1-q}^{2}(\Omega)^{40}$ depend on the orientation of the $I-S$ dipoledipole coupling with respect to the laboratory frame, described by the $\Omega$ angle (for simplicity it has been assumed that the dipole-dipole axis and the EFG tensor axis coincide) and $\mathrm{r}$ is interspin distance. The expressions of Eqs. (A1) and (A2) are equivalent to the typically used form of the dipole-dipole Hamiltonian: $H_{D D}(I, S, \Omega)=\sqrt{6}\left(\frac{\mu_{0}}{4 \pi} \frac{\gamma_{I} \gamma_{S} \hbar}{r_{I S}^{3}}\right) \sum_{q=-2}^{2}(-1)^{q} D_{0,-q}^{2}(\Omega) T_{q}^{2}(I, S)$. This representation can also be used for the quadrupole Hamiltonian $H_{Q}(S, \Omega)$ expressed in the laboratory frame:

$$
H_{Q}(S)=\frac{1}{2} \sqrt{\frac{3}{2}} \frac{a_{Q}}{S(2 S-1)} \sqrt{\frac{(2 S+3)(2 S+1)(S+1)(2 S-1)}{30}} \sum_{q=-2}^{2}(-1)^{q}\left[D_{0,-q}^{2}(\Omega)+\frac{\eta}{\sqrt{6}}\left(D_{2,-q}^{2}(\Omega)+D_{-2,-q}^{2}(\Omega)\right)\right] S_{q}^{2}(\mathrm{~A}
$$

The $S_{q}^{2}$ operators are related to the $T_{q}^{2}(S)$ components as: $S_{q}^{2}=\sqrt{\frac{30}{(2 S+3)(2 S+1)(S+1)(2 S-1)}} T_{q}^{2}(S)$ $\left(T_{0}^{2}(S)=\frac{1}{\sqrt{6}}\left[3 S_{z}^{2}-S(S+1)\right], T_{ \pm 1}^{2}(S)=\mp \frac{1}{2}\left[S_{z} S_{ \pm}+S_{ \pm} S_{z}\right]\right.$ and $\left.T_{ \pm 2}^{2}(S)=\frac{1}{2} S_{ \pm} S_{ \pm}\right)$. The $S$ spin variables (the spin quantum number $S$ and the magnetic spin quantum number $m$ ) lead to a Liouville basis $\{S, m\rangle\langle S m|\}$ constructed from pairs of the Zeeman functions, $|S, m\rangle$. The tensor components $S_{q}^{1}$ and $S_{q}^{2}$ can be expressed in the Liouville basis as: $1,2,8-13,19-21,35-38$ 


$$
\left.S_{\sigma}^{\Sigma}=\mid \Sigma, \sigma\right)=\sum_{m=-S}^{S}(-1)^{S-m-\sigma} \sqrt{2 \Sigma+1}\left(\begin{array}{ccc}
S & S & \Sigma \\
m+\sigma & -m & -\sigma
\end{array}\right)|S, m+\sigma\rangle\langle S, m|
$$

As the spin and spacial variables cannot be separated in the stochastic Liouville approach one constructs a basis being an outer product of the spin and rotational variables: 1,2,8-13,19-21,35-38

$\left.\left.\left.O_{\alpha}\right)=\mid \Sigma, \sigma\right) \otimes \mid L, K, M\right)$

where $\Sigma$ ranges from 1 to $(2 S+1)$, while the rotational vector is defined as:

$$
L, K, M)=\frac{\sqrt{2 L+1}}{4 \pi} D_{K, M}^{L}(\Omega)
$$

The ${ }^{1} \mathrm{H}$ spin-lattice relaxation rate is given as a matrix product: ${ }^{1,2,8-13,19-21,35-38}$

$$
R_{1}=\operatorname{Re}\left\{\left[T_{1}^{1, D D}\right]^{+}[M]^{-1}\left[T_{1}^{1, D D}\right]\right\}
$$

where the matrix $[M]$ is defined in the $\left\{O_{\alpha}\right)\left(O_{\beta} \mid\right\}$ basis constructed from pairs of the $\left.\mid O_{\alpha}\right)$ vectors (the vectors $\left.\mid O_{\alpha}\right)$ and $\left.O_{\beta}\right)$ are given as $\left.\left.\mid \Sigma, \sigma\right) \otimes \mid L, K, M\right)$ and $\left.\left.\mid \Sigma^{\prime}, \sigma^{\prime}\right) \otimes \mid L^{\prime}, K^{\prime}, M^{\prime}\right)$, respectively). The matrix elements are given as:

$$
\begin{aligned}
& {[M]_{\alpha, \beta}=\frac{1}{4 S(2 S-1)} a_{Q}(-1)^{\sigma^{\prime}} F_{\left|K-K^{\prime}\right|}^{2}\left[(-1)^{\Sigma^{\prime}+\Sigma}-1\right] \times} \\
& \sqrt{(2 S+3)(2 S+1)(S+1) S(2 S-1)\left(2 L^{\prime}+1\right)(2 L+1)\left(2 \Sigma^{\prime}+1\right)(2 \Sigma+1)} \times \\
& \left(\begin{array}{ccc}
L^{\prime} & 2 & L \\
-K^{\prime} & K^{\prime}-K & K
\end{array}\right)\left(\begin{array}{ccc}
L^{\prime} & 2 & L \\
-M^{\prime} & M^{\prime}-M & M
\end{array}\right)\left(\begin{array}{ccc}
\Sigma^{\prime} & 2 & \Sigma \\
-\sigma^{\prime} & B^{\prime}-B & \sigma
\end{array}\right)\left\{\begin{array}{ccc}
\Sigma^{\prime} & 2 & \Sigma \\
S^{\prime} & S & S
\end{array}\right\}+ \\
& \delta_{L L^{\prime}} \delta_{K K^{\prime}} \delta_{M M^{\prime}} \delta_{\Sigma \Sigma^{\prime}} \delta_{\sigma \sigma^{\prime}}\left(\omega_{S} \sigma+\frac{i L(L+1)}{6 \tau_{\text {rot }}}\right)
\end{aligned}
$$

where $F_{0}^{1}=1, F_{1}^{1}=0, F_{2}^{1}=\frac{\eta}{\sqrt{6}}$.

The matrix $\left[T_{1}, D D\right]$ is a representation of $T_{1}^{1, D Q}(S)$ in the basis $\left\{\left(O_{\alpha}\right)\right\}$ and it contains only three nonzero elements) associated with the functions: $\mid 1,-1) \otimes \mid 2,0,2), \mid 1,0) \otimes(2,0,1)$ and $|1,1| \otimes \mid 2,0,0)$; the elements are equal to $\left(\begin{array}{ccc}1 & 2 & 1 \\ -1 & 2 & -1\end{array}\right),\left(\begin{array}{ccc}1 & 2 & 1 \\ 0 & 1 & -1\end{array}\right)$ and $\left(\begin{array}{ccc}1 & 2 & 1 \\ 1 & 0 & -1\end{array}\right)$, respectively $(1 / 5,1 / 10$, 1/30). ${ }^{1,2,8-13,19-21,35-38}$ 
In the presence of exchange dynamics the diagonal term $\left(\omega_{S} \sigma+\frac{i L(L+1)}{6 \tau_{\text {rot }}}\right)$ should be extended to the form: $\left(\omega_{S} \sigma+\frac{i L(L+1)}{6 \tau_{\text {rot }}}+\frac{1}{\tau_{e x}}\right)$, where $\tau_{e x}$ denotes the exchange life time. 


\section{Appendix B}

\section{Exponentiality of ${ }^{1} \mathrm{H}$ spin-lattice relaxation in $\mathrm{THF}$ solution of tris(2- methoxyphenyl)bismuthane - magnetisation curves}

Figure $4(\mathrm{a}$ and $\mathrm{b}$ ) shows normalized magnetization curves for selected frequencies at $185 \mathrm{~K}$, $200 \mathrm{~K}, 253 \mathrm{~K}$ and 293K. Solid lines show monoexponential fits.

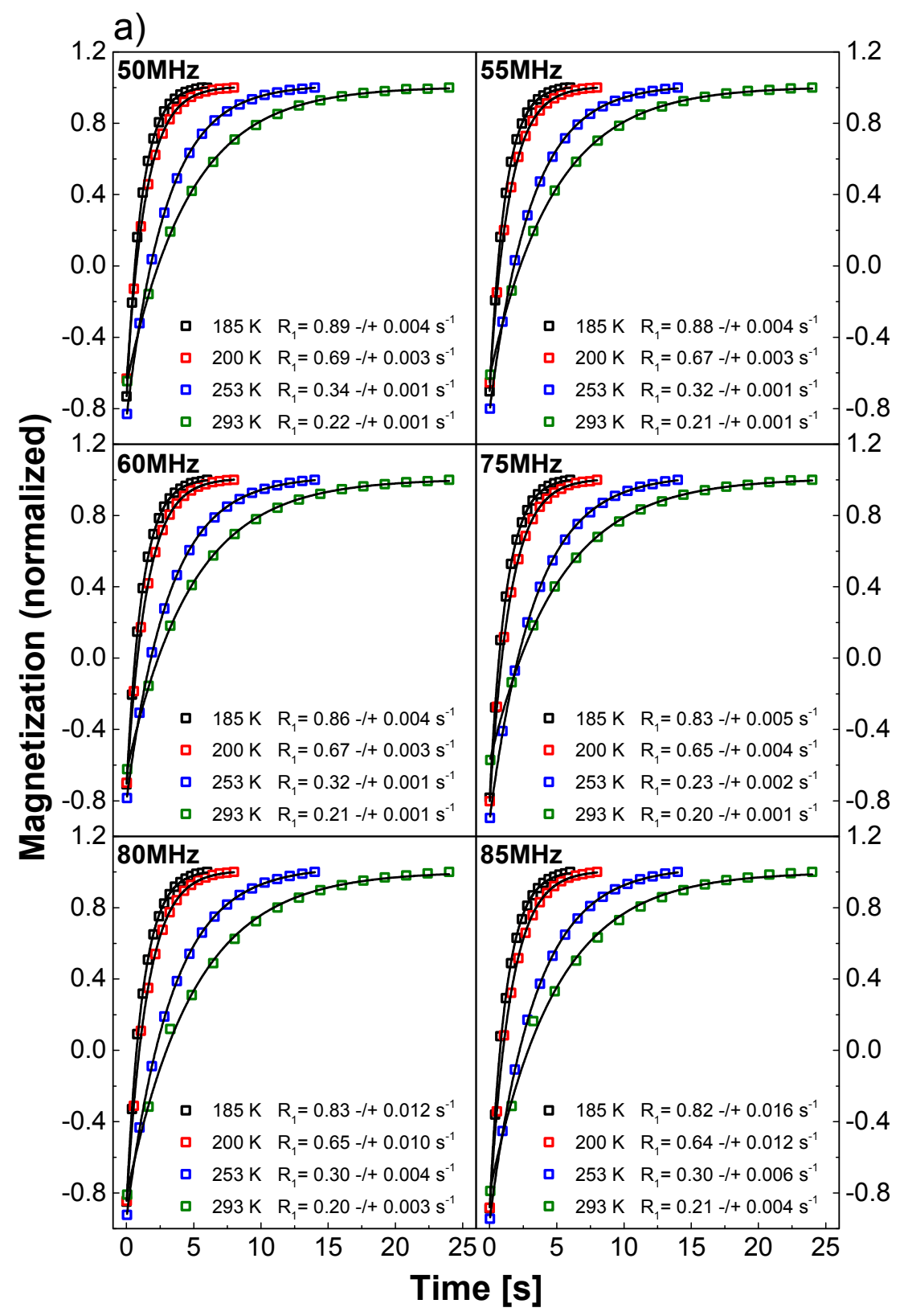




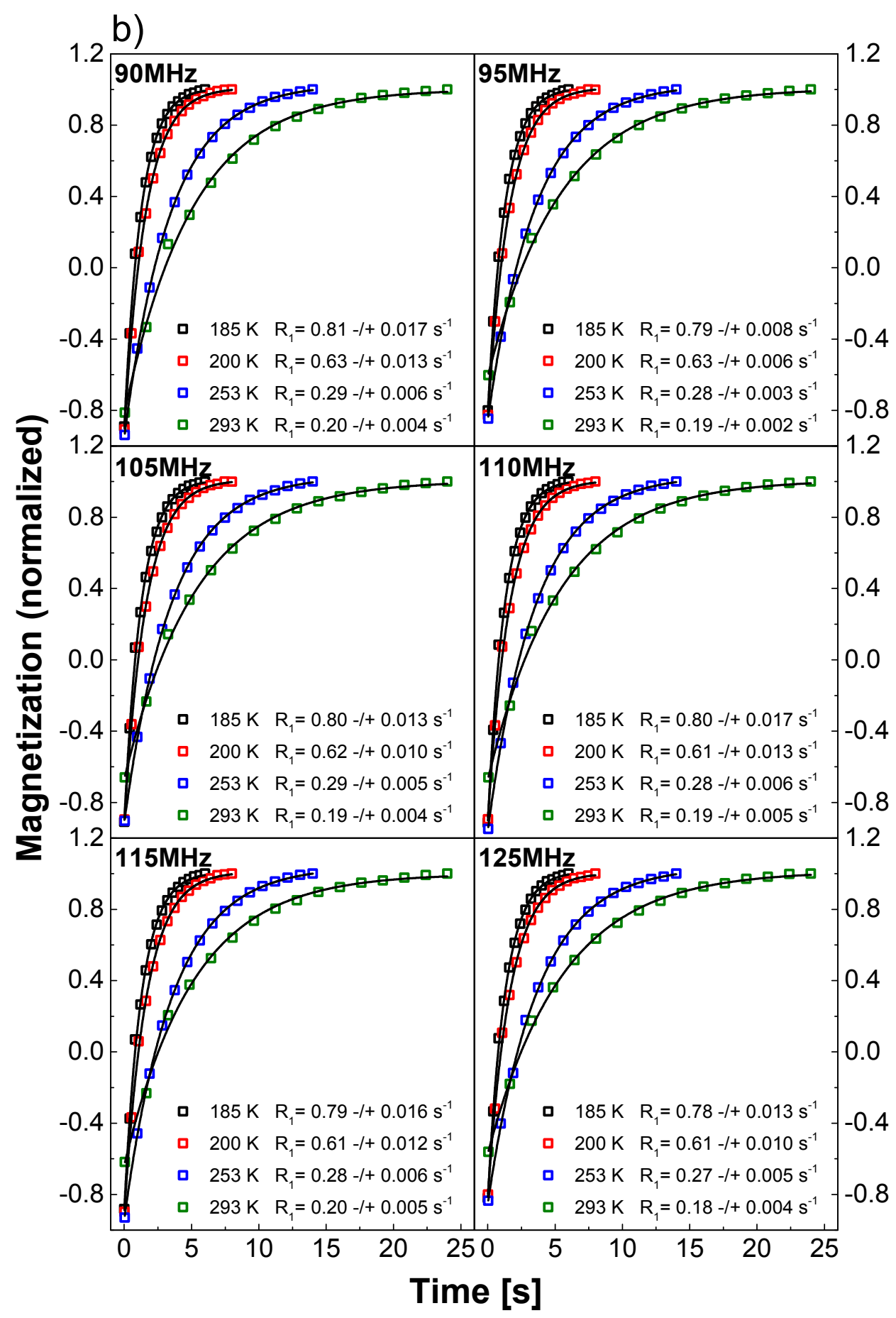

FIG. 4. ${ }^{1} \mathrm{H}$ magnetization versus time for tris(2-methoxyphenyl)bismuthane in THF. Solid lines show single exponential fits. 
${ }^{1} \mathrm{H}$ spin-lattice relaxation rates for pure THF

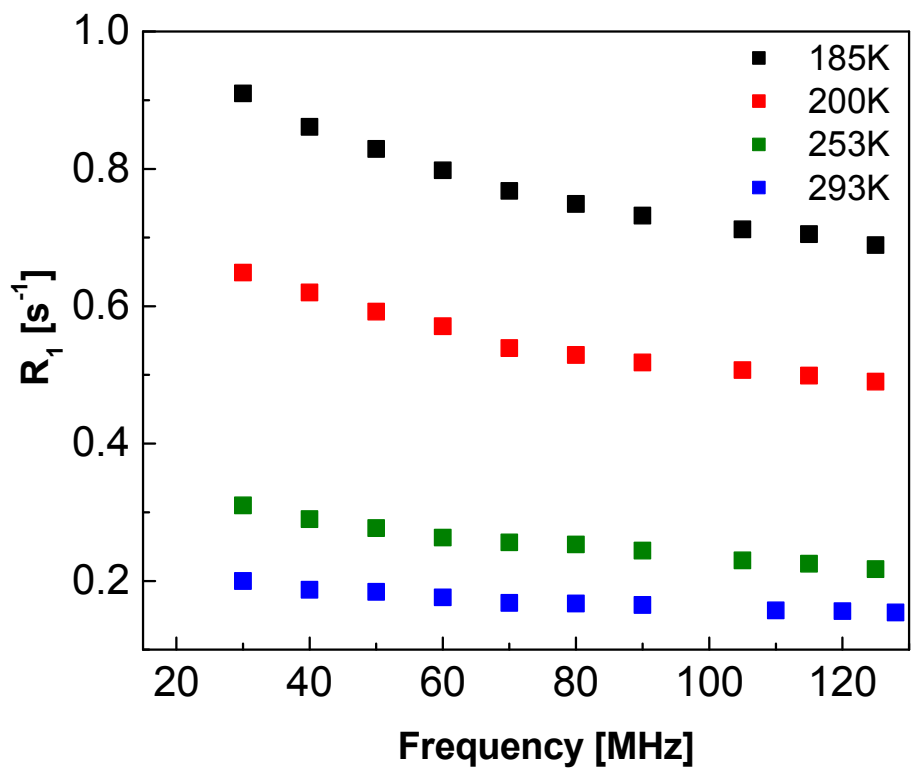

FIG. 5. ${ }^{1} \mathrm{H}$ spin-lattice relaxation rates of pure THF. 
